\title{
Time-Resolved Measurements of Suprathermal Ion Transport Induced by Intermittent Plasma Blob Filaments
}

\author{
A. Bovet, ${ }^{*}$ A. Fasoli, and I. Furno \\ École Polytechnique Fédérale de Lausanne (EPFL), Centre de Recherches en Physique des Plasmas (CRPP), \\ CH-1015 Lausanne, Switzerland \\ (Received 26 August 2014; published 26 November 2014)
}

\begin{abstract}
Suprathermal ion turbulent transport in magnetized plasmas is generally nondiffusive, ranging from subdiffusive to superdiffusive depending on the interplay of the turbulent structures and the suprathermal ion orbits. Here, we present time-resolved measurements of the cross-field suprathermal ion transport in a toroidal magnetized turbulent plasma. Measurements in the superdiffusive regime are characterized by a higher intermittency than in the subdiffusive regime. Using conditional averaging, we show that, when the transport is superdiffusive, suprathermal ions are transported by intermittent field-elongated turbulent structures that are radially propagating.
\end{abstract}

DOI: 10.1103/PhysRevLett.113.225001

PACS numbers: 52.25.Gj, 05.45.Tp, 52.25.Fi, 52.55.Pi

Understanding turbulent transport of suprathermal ions, i.e., ions with energies greater than the quasi-Maxwellian background plasma, is of paramount importance for a variety of laboratory and natural systems. In future fusion reactors such as ITER and DEMO, a good confinement of suprathermal ions, created by fusion reactions or additional heating, is necessary to reach and control burning plasma conditions $[1,2]$. In astrophysical plasmas, the understanding of solar energetic particles and of cosmic ray transport still has some gray areas [3,4]. For example, during impulsive solar energetic particle events, large fluctuations in intensity, called dropouts, were observed and are not fully understood [5].

Experimental and numerical evidence suggests that suprathermal ion cross-field transport can be nondiffusive [6-10], characterized by a variance of particle displacements that does not scale linearly with time $\left\langle[\boldsymbol{r}(t)-\boldsymbol{r}(0)]^{2}\right\rangle \propto t^{\gamma}$, with the transport exponent $\gamma \neq 1$ generally. Here, $\boldsymbol{r}(t)$ indicates the particle positions. For $0<\gamma<1$ the transport is subdiffusive, for $1<\gamma<2$ superdiffusive. Classical diffusion corresponds to $\gamma=1$. In fusion experiments and astrophysical plasmas, measurements are limited to a few positions by high-temperature and diagnostics accessibility. In those cases, it is not possible to characterize the transport by the temporal evolution of the variance of displacements and information about the transport has to be inferred from the time trace statistics [11-13].

In this Letter, we present first time-resolved measurements of the cross-field transport of suprathermal ions in a turbulent magnetized plasma. Previously, in the TORPEX device [14], by using three-dimensional time-averaged measurements of the width of a suprathermal ion beam in combination with numerical simulations (an example is shown in Fig. 1), we have shown that the transport of suprathermal ions varies from superdiffusive to subdiffusive as their energy is increased [8,10,15-19]. We consider here two suprathermal ion energies, 30 and $70 \mathrm{eV}$, for which the radial transport was identified to be superdiffusive $(\gamma=1.20)$ and subdiffusive $(\gamma=0.51)$, respectively $[10,18]$. We show that the time traces of the suprathermal ion current show a clear difference in intermittency. Using the technique of conditional average sampling (CAS) [20,21], we identify the effect of turbulent structures on the suprathermal ion beam confirming the efficiency of gyroaveraging, depending on the ion energy, to decrease transport.

TORPEX (Fig. 1) has a major radius $R=1 \mathrm{~m}$ and a minor radius $a=20 \mathrm{~cm}$. A toroidal magnetic field $B_{t}=$ $74 \mathrm{mT}$ and a weaker vertical magnetic $B_{v}=2 \mathrm{mT}$ create open helical magnetic field lines that terminate on the top and bottom of the vessel, resulting in a vertically elongated

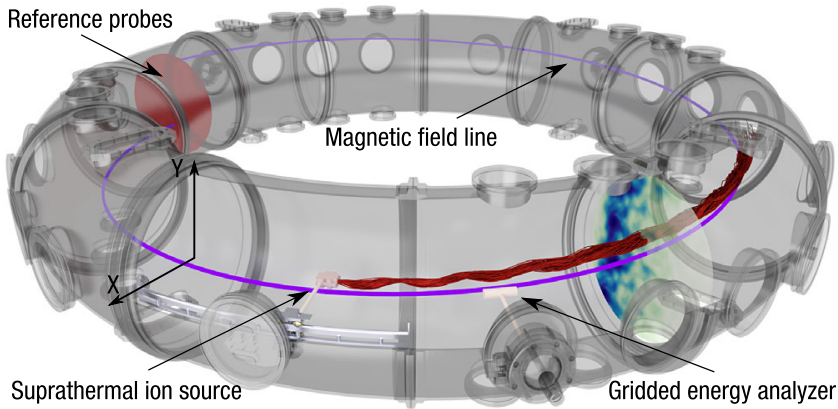

FIG. 1 (color online). Experimental setup showing the TORPEX device. The suprathermal ion source and the gridded energy analyzer are shown. The poloidal plane in red indicates the position of the reference probes used for the CAS. A helical magnetic field line is shown in violet. The field lines crossing the suprathermal ion beam between the source and the detector cross the reference probe plane twice. Examples of computed suprathermal ion trajectories are shown in red along with a snapshot of the electric potential fluctuations as simulated by the GBS code [10,22]. 
plasma configuration. In the present experiments, microwaves in the electron cyclotron range of frequencies are used to produce and sustain hydrogen plasmas. We use a coordinate system centered in the middle of the poloidal section to measure positions in the poloidal plane as shown in Fig. 1. The region of plasma production and an idealinterchange mode [23], characterized by parallel and vertical wave numbers $k_{\|} \simeq 0$ and $k_{v} \simeq 33 \mathrm{rad} / \mathrm{m}$, are located in the high-field side of the device $(X \simeq-12 \mathrm{~cm})$. The vertical wavelength of the mode is equal to the magnetic field line return distance, $\Delta=2 \pi R\left(B_{v} / B_{t}\right) \simeq 18.8 \mathrm{~cm}$. The turbulence in the region defined by $X \gtrsim-5 \mathrm{~cm}$ is characterized by the presence of field-elongated structures, termed "blobs," intermittently generated by the idealinterchange mode and propagating radially outwards in the low-field side $[24,25]$.

A miniaturized $\mathrm{Li}^{6}$ ion source (Fig. 1), positioned at $X=1.5$ and $Y=-4.3 \mathrm{~cm}$, in the region dominated by blobs, injects suprathermal ions in the toroidal direction. At the injection position the time-averaged electron temperature measured by a triple probe [26] is $T_{e} \simeq 1.3 \mathrm{eV}$. A bias voltage applied to the emitter accelerates the ions to the desired energy. In the absence of a plasma, the motion of the ions is a combination of their gyromotion along the magnetic field lines and their vertical drift due to the curvature and gradient of $\mathbf{B}$. Measurements at different poloidal locations are taken using a gridded energy analyzer (GEA) [18] situated at a distance $d=41.2 \mathrm{~cm}$ along the toroidal axis. At this distance, suprathermal ions have completed approximately two gyro-orbits and their interaction with the turbulence is most important $[8,10]$. The GEA has an inlet diameter of $8 \mathrm{~mm}$ and is used to selectively measure the current produced by ions having an energy larger than $10 \mathrm{eV}$, repelling electrons. The signals are digitized at $250 \mathrm{kHz}$.

Figure 2 shows two examples of suprathermal ion current time traces from the GEA for ion energies of 30 and $70 \mathrm{eV}$. The bias voltage on the emitter is turned on and off at $\sim 30 \mathrm{~Hz}$ allowing us to differentiate the fluctuations of the background noise from those of the suprathermal ion current. During the on phase, the time trace for $30 \mathrm{eV}$ ions shows a higher intermittency [Fig. 2(a)] than the $70 \mathrm{eV}$ case [Fig. 2(b)]. This difference is reflected in the probability distribution functions (PDFs) of the current fluctuations of the two cases (Fig. 2 insets). The PDF for the $30 \mathrm{eV}$ case is positively skewed reflecting the fact that the suprathermal ions reach the detector in bursts, while the PDF for the $70 \mathrm{eV}$ ions does not show large fluctuations.

Figure 3 displays two-dimensional profiles of the timeaveraged suprathermal ions current density for the two energies. The periods during which the source is not emitting are used to compute the position-dependent background values of the mean (offset) and skewness. These are removed from the values computed during the on phases. Although the time-averaged profiles for the two energies
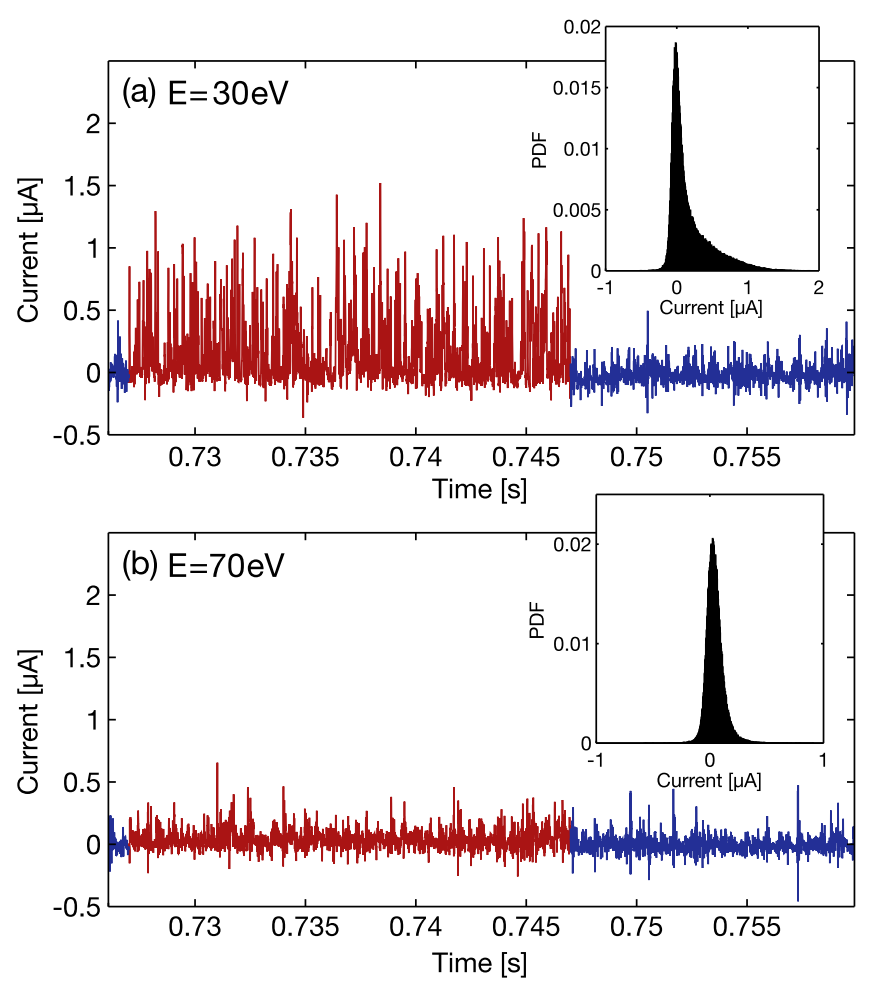

FIG. 2 (color online). Suprathermal ion current time traces measured with the GEA positioned at the locations indicated by the black crosses in Fig. 3. The suprathermal ion source is turned on (red) and off (blue) periodically. The time trace of $30 \mathrm{eV}$ ions (a) shows a high intermittency level contrary to the $70 \mathrm{eV}$ ions time trace (b). Insets show the PDFs of the suprathermal current fluctuations. The PDF for the $30 \mathrm{eV}$ case is strongly positively skewed, reflecting the intermittency of the signal, whereas the PDF for the $70 \mathrm{eV}$ case is not.

are relatively similar, when the skewness profile of the time traces is reconstructed [Figs. 3(c) and 3(d)], a clear difference appears. While the skewness profile for the $70 \mathrm{eV}$ ion beam is flat, the profile for the $30 \mathrm{eV}$ ions reveals a region of high skewness around the peak of maximum timeaverage current. This implies that, in the surrounding of the profile, where the time traces have a low time-averaged current compared to the center of the profile, the intermittency is more important. In the following, we will show that this intermittency is caused by the interaction of the suprathermal ions with blobs.

To identify the effect of blobs on the suprathermal ion beam we use the CAS over many blob events. To detect blobs, we use the Langmuir probe array HEXTIP [27] situated at a toroidal angle of $240^{\circ}$ away from the detector (Fig. 1). Blobs are defined as bursts in the ion saturation current characterized by $n \sigma<\tilde{I}_{\text {sat }}<m \sigma$, where $\tilde{I}_{\text {sat }}=$ $I_{\text {sat }}-\bar{I}_{\text {sat }}$ is the ion saturation current fluctuations, $\sigma$ is the standard deviation of the ion saturation current signal and, $n$ and $m$ are factors allowing us to select the amplitude of events in a certain range. The suprathermal ion current is averaged in a time window centered around each blob 


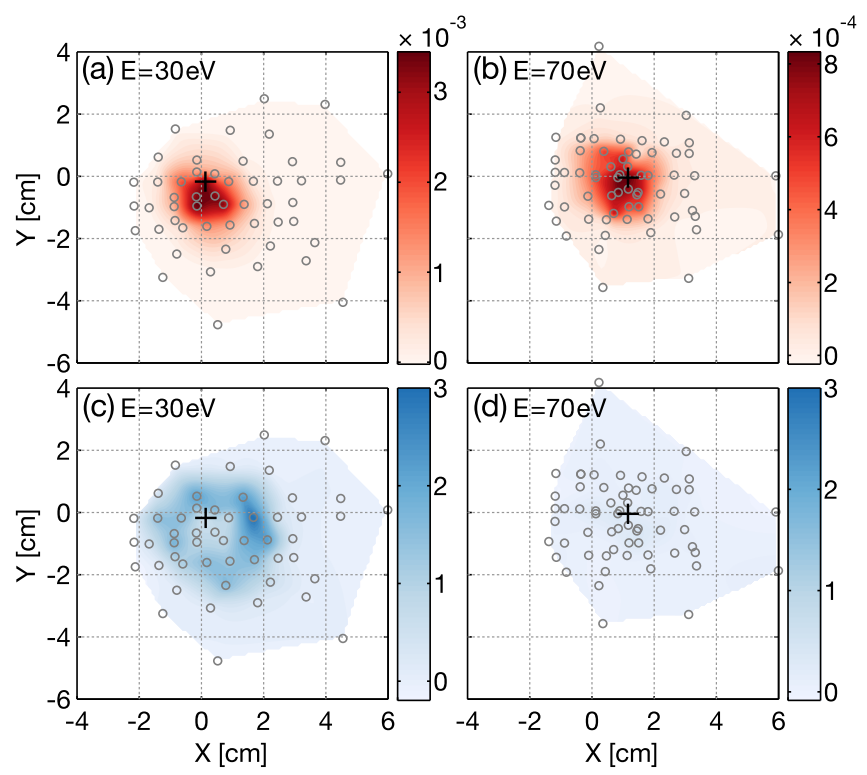

FIG. 3 (color online). Poloidal profiles of the time-averaged suprathermal ion current density, in $\mathrm{A} / \mathrm{m}^{2}$, for ions at 30 (a) and $70 \mathrm{eV}$ (b) and of the skewness (c) and (d). The profile of the skewness of the $30 \mathrm{eV}$ ions (c) reveals a crown of high skewness around the peak of the time-average profile. This indicates that the broadening of the $30 \mathrm{eV}$ suprathermal ion beam is due to intermittent bursts perturbing the gyromotion of the ions. This pattern is not visible on the skewness profile for $70 \mathrm{eV}$ ions (d). Gray circles show the positions of measurements.

event. This procedure is applied to the phase with and without suprathermal ions. The difference between the two CAS shows the dynamic of the suprathermal ion beam when a blob is detected on a given probe tip of HEXTIP.

Figure 4 shows the time evolution of the CAS suprathermal ion current profile for the two energies, 30 and $70 \mathrm{eV}$ (see Supplemental Material [28] for a movie showing the same data). The reference probe is positioned at $X=0$ and $Y=12.1 \mathrm{~cm}$ and all events exceeding a level $n=3$ are selected. Because of the helical magnetic configuration, blobs detected on this tip are aligned to a magnetic field line that is situated at the position $X=0, Y=-0.5 \mathrm{~cm}$ at the toroidal location of the detector, which is close to the maximum of the time-averaged profiles [Figs. 3(a) and 3(b)]. Figures 4(a) and 4(b) show the profiles $40 \mu$ s before the detection of the blob, a duration corresponding approximately to the autocorrelation time of floating potential fluctuations in the blob region $\left(\tau_{\mathrm{corr}, V_{\mathrm{ff}}} \simeq 42 \mu \mathrm{s}\right)$ and of $I_{\mathrm{sat}}$ fluctuations $\left(\tau_{\mathrm{corr}, I_{\mathrm{sat}}} \simeq 52 \mu \mathrm{s}\right)$. The effect of the blob as it passes through the $30 \mathrm{eV}$ ion beam is revealed in Figs. 4(a), 4(c), and 4(e). First, the beam is displaced to the left with respect to its time-averaged profile (gray contours) [Fig. 4(a)]. Then, when the blob is detected on the probe [Fig. 4(c)], the displacement changes from left to right. At later times [Fig. 4(e)], the beam is displaced to the bottom right of its averaged position. The time scale of the beam displacement is comparable to the duration of the
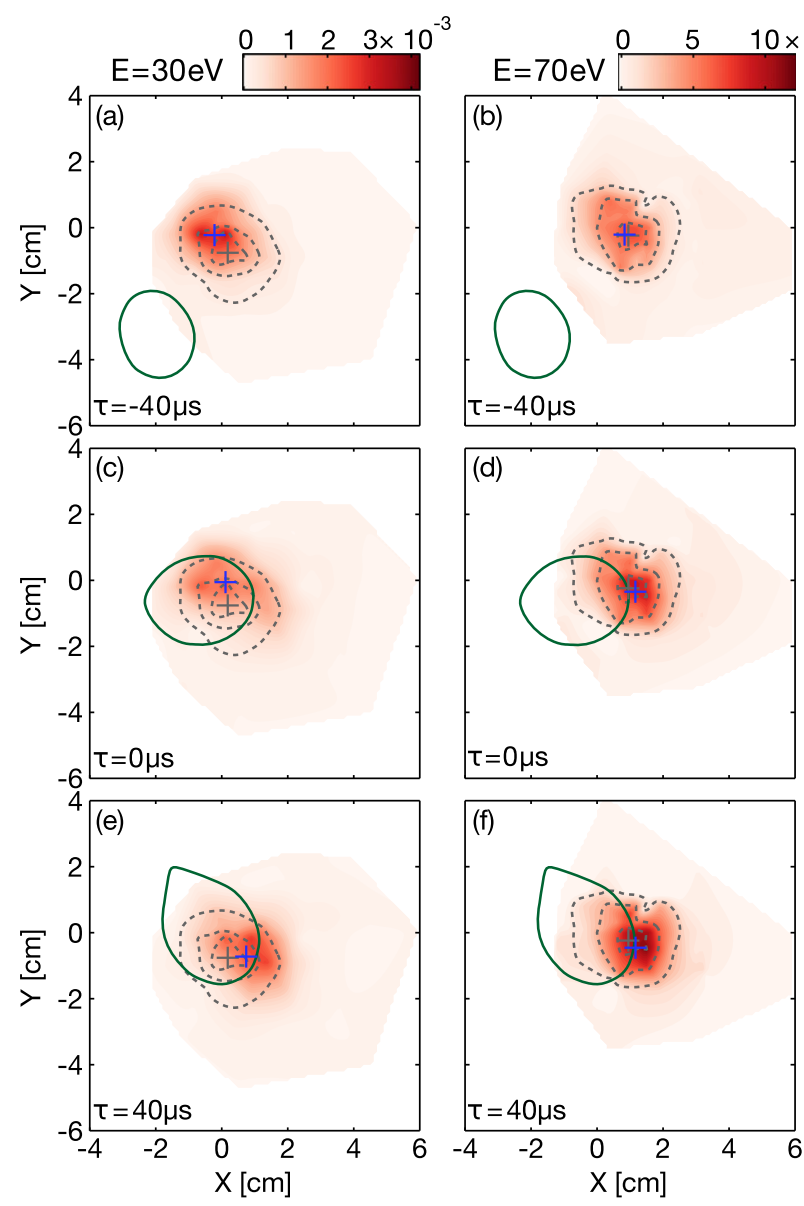

FIG. 4 (color online). Conditionally averaged dynamics of the suprathermal ion current density $\left[\mathrm{A} / \mathrm{m}^{2}\right]$, for the $30 \mathrm{eV}$ case (left) and the $70 \mathrm{eV}$ case (right) at three different times. Dashed gray contours represent time-average profiles (Fig. 3) and the green contour represents the averaged blob $I_{\text {sat }}$ profile. The center of mass positions of the beam and of the time-averaged profiles are indicated by blue and gray crosses, respectively. The effect of the blob, as it passes through the suprathermal ion beam, is much more important for $30 \mathrm{eV}$ ions than for $70 \mathrm{eV}$ ions.

intermittent bursts shown in Fig. 2(a). This is demonstrated by the value of the autocorrelation time $\left(\tau_{\text {corr }} \simeq 22 \mu \mathrm{s}\right)$ of the most intermittent suprathermal ions time traces (with a skewness above 2) which is comparable to the $I_{\text {sat }}$ autocorrelation time. Figures 4(b), 4(d), and 4(f) show that as the same CAS blob crosses the $70 \mathrm{eV}$ ion beam, the displacement of the beam is extremely small compared to the one of the $30 \mathrm{eV}$ beam. See Supplemental Material at [28] for a movie of the same analysis but with a blob passing far away from the beam (the reference probe position is $X=10.5, Y=0 \mathrm{~cm}$ ) showing that, in this case, the beam is not perturbed.

As the time of flight between the source and the detector is smaller for $70 \mathrm{eV}$ ions $\left(t_{f, 70 \mathrm{eV}} \simeq 9 \mu \mathrm{s}\right)$ than for $30 \mathrm{eV}$ ions $\left(t_{f, 30 \mathrm{eV}} \simeq 13 \mu \mathrm{s}\right)$, a smaller perpendicular displacement is expected for $70 \mathrm{eV}$ ions. The maximum displacement of the center of mass of the $30 \mathrm{eV}$ ion beam is 
$d_{\perp .30 \mathrm{eV}} \simeq 0.8$ and $d_{\perp, 70 \mathrm{eV}} \simeq 0.3 \mathrm{~cm}$ for the $70 \mathrm{eV}$ ion beam. Considering the simplified picture of the effect of a blob as inducing a perpendicular displacement at a constant velocity of the guiding center of the ions, the perpendicular displacement is given by $d_{\perp}=v_{\perp} t_{f}$, where $v_{\perp}=\tilde{E} / B$ and $\tilde{E}$ is the electric field of the blob. Since the electric field is the same in both cases, we should have $d_{\perp, 30 \mathrm{eV}} / d_{\perp, 70 \mathrm{eV}}=$ $t_{f, 30 \mathrm{eV}} / t_{f, 70 \mathrm{eV}}$. Instead, we find $d_{\perp, 30 \mathrm{eV}} / d_{\perp, 70 \mathrm{eV}} \simeq 2.7>$ $1.4 \simeq t_{f, 30 \mathrm{eV}} / t_{f, 70 \mathrm{eV}}$. This indicates a weaker interaction between suprathermal ions and blobs in the $70 \mathrm{eV}$ case than in the $30 \mathrm{eV}$ case, consistent with the absence of intermittency in the current time traces in Fig. 2(b) and Fig. 3(d), and with the increased effect of gyroaveraging for ions having larger Larmor radii [8]. The value of the electric field estimated from the displacement of the $30 \mathrm{eV}$ ion beam is $\tilde{E} \simeq 45 \mathrm{~V} / \mathrm{m}$, which is comparable to previously measured values $[29,30]$.

To investigate the mechanisms perturbing the trajectories of suprathermal ions, we study the effect of blobs at different positions in the poloidal plane. Using probe tips at different vertical positions between the radial position $0 \mathrm{~cm} \leq X \leq 1.7 \mathrm{~cm}$ as reference probes for the CAS, we compute the horizontal and vertical displacement of the beam center of mass as a function of time. We separate the blobs in two classes depending on the amplitude of their $I_{\text {sat }}$ fluctuations: small blobs with $2 \sigma<\tilde{I}_{\text {sat }}<4 \sigma$ and large blobs with $\tilde{I}_{\text {sat }}>4 \sigma$.

Figure 5 shows the horizontal and vertical displacement for 30 and $70 \mathrm{eV}$ ions due to large blobs. The circles represent the position of the suprathermal ion source projected along the helical magnetic field lines on the poloidal plane of the reference probes. The crosses represent the position of the beam center, when it reaches the detector, projected onto the same plane. Thus, the vertical distance between the circles and the crosses shows the vertical distance traveled by the ions between their emission and their detection due to the vertical drift. The error on the displacement is estimated by simulating the measurements on prescribed profiles and is $\pm 1 \mathrm{~mm}$. We observe that $30 \mathrm{eV}$ ions are systematically more displaced by the blobs than $70 \mathrm{eV}$ ions. Figure 5 reveals also that, depending on the position of the blob compared to its position, the beam can be displaced inwards or outwards. We note that, the beams are not displaced when a blob transits far away from them (for probes located at $Y \simeq 0 \mathrm{~cm}$ ). We observe that blobs passing above the beam tend to move it outwards and that blobs passing below it tend to move it inwards. The plasma potential associated with blobs in TORPEX has been identified with a bipolar structure that results from the $\nabla B$ and curvature induced charge separation inside blobs [25]. The observed suprathermal ion beam displacement is consistent with the bipolar potential structure, taking into account that in the present plasma conditions the blob motion can have a strong vertical component. The same study for lower
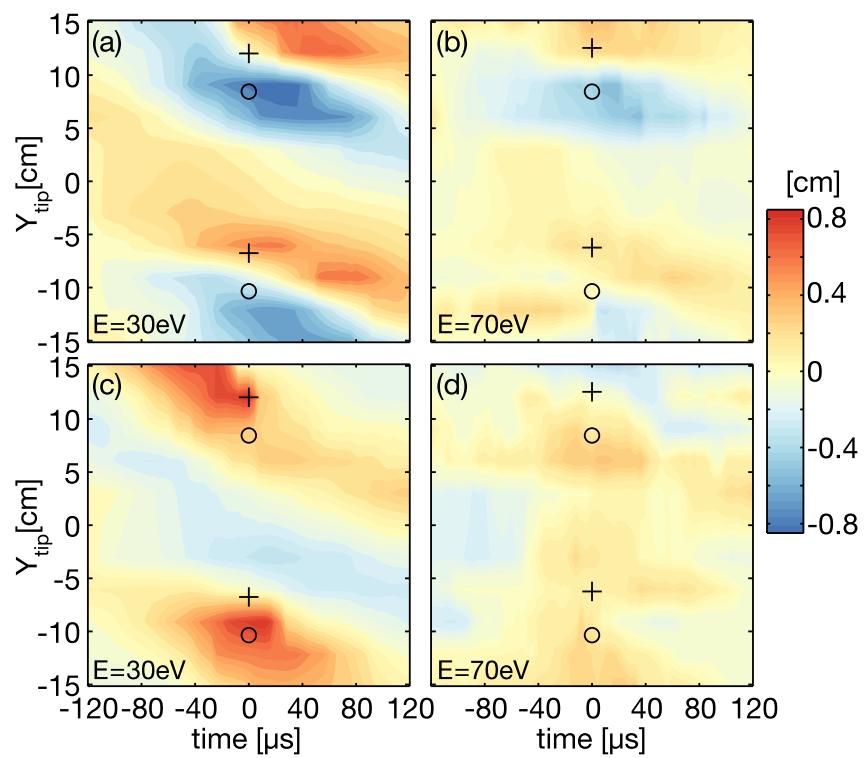

FIG. 5 (color online). Horizontal (top row) and vertical (bottom row) displacement of the suprathermal ion beam due to large blobs $\left(\tilde{I}_{\text {sat }}>4 \sigma\right)$ for 30 and $70 \mathrm{eV}$ suprathermal ions. On all panels, the $x$ axis represents the time relative to the detection of the blob on the reference probe and the $y$ axis represents the vertical position of the reference probe. The crosses and the circles indicate the positions of the beam center, when it reaches the detector, and of the source, respectively, projected along the helical magnetic field lines on the poloidal plane of the reference probes (see Fig. 1). The measurement error is $\pm 1 \mathrm{~mm}$. The vertical periodicity of the magnetic field is reflected in the pattern with a field line return distance of $\Delta \simeq 18.8 \mathrm{~cm}$.

amplitude blobs reveals the same pattern of displacements with amplitudes smaller by about a factor of 2 .

In summary, thanks to unprecedented time-resolved measurements, we show that suprathermal ions in TORPEX plasmas experiencing superdiffusive transport are associated with bursty displacement events resulting in highly intermittent time traces when detected. Suprathermal ions experiencing subdiffusive transport do not display such intermittency. The intermittency of the time traces is quantified by the skewness of their fluctuations. For superdiffusive ions, the skewness poloidal profile reveals a crown around the peak of maximum current, where the fluctuations are the most intermittent. Using CAS measurements, we prove that the intermittency in the superdiffusive ions is due to their higher sensitivity to intermittent blobs, which move the ions through their electrical field both inwards and outwards depending on their relative location. Larger blobs have a greater effect than smaller blobs.

Our work links observations usually unaccessible in fusion devices and astrophysical plasmas, namely, energyresolved three-dimensional time-averaged measurements, with Eulerian time-resolved measurements, which are often the only accessible measurements in such experiments. The 
presence of large fluctuations in the time traces of superdiffusive ions corroborates our previous theoretical study, where we showed that Lévy, heavy-tailed, statistics govern this regime $[16,19]$. In burning plasmas, strong intermittency in the transport of suprathermal ions can be a concern, because of possible effects on the core reactivity, and, on the heat load that the suprathermal ions may produce on the reactor first wall even in situations of negligible average loss rates.

The authors would like to thank F. Avino, J. Loizu, and P. Ricci for their helpful discussions and C. Theiler for his careful reading of the manuscript and suggestions to improve it. Fruitful discussions with members of the team number 297 "Superdiffusive Transport in Space Plasmas and its Influence on Energetic Particle Acceleration and Propagation" at the ISSI are acknowledged. This work was supported in part by the Swiss National Science Foundation.

*alexandre.bovet@epfl.ch

[1] A. Fasoli et al., Nucl. Fusion 47, S264 (2007).

[2] D. Zarzoso et al., Phys. Rev. Lett. 110, 125002 (2013).

[3] R. B. Decker, S. M. Krimigis, E. C. Roelof, M. E. Hill, T. P. Armstrong, G. Gloeckler, D. C. Hamilton, and L. J. Lanzerotti, Nature (London) 454, 67 (2008).

[4] D. Perrone, R. O. Dendy, I. Furno, R. Sanchez, G. Zimbardo, A. Bovet, A. Fasoli, K. Gustafson, S. Perri, P. Ricci, and F. Valentini, Space Sci. Rev. 178, 233 (2013).

[5] F. Guo and J. Giacalone, Astrophys. J. 780, 16 (2014).

[6] S. Perri and G. Zimbardo, Adv. Space Res. 44, 465 (2009).

[7] S. Zhou, W. W. Heidbrink, H. Boehmer, R. McWilliams, T. Carter, S. Vincena, S. K. P. Tripathi, P. Popovich, B. Friedman, and F. Jenko, Phys. Plasmas 17, 092103 (2010).

[8] K. Gustafson, P. Ricci, I. Furno, and A. Fasoli, Phys. Rev. Lett. 108, 035006 (2012).

[9] W. W. Heidbrink et al., Plasma Phys. Controlled Fusion 54, 124007 (2012).

[10] A. Bovet, A. Fasoli, K. Gustafson, P. Ricci, and I. Furno, [Phys. Rev. Lett. (to be published)].
[11] B. A. Carreras, V. E. Lynch, D. E. Newman, R. Balbn, J. Bleuel, M. a. Pedrosa, M. Endler, B. van Milligen, E. Sanchez, and C. Hidalgo, Phys. Plasmas 7, 3278 (2000).

[12] N. Scafetta and B. J. West, Phys. Rev. Lett. 90, 248701 (2003).

[13] M. Rajković, M. Škorić, K. Sø lna, and G. Antar, Nucl. Fusion 48, 024016 (2008).

[14] A. Fasoli et al., Nucl. Fusion 53, 063013 (2013).

[15] K. Gustafson, P. Ricci, A. Bovet, I. Furno, and A. Fasoli, Phys. Plasmas 19, 062306 (2012).

[16] K. Gustafson and P. Ricci, Phys. Plasmas 19, 032304 (2012).

[17] A. Bovet, I. Furno, A. Fasoli, K. Gustafson, and P. Ricci, Nucl. Fusion 52, 094017 (2012).

[18] A. Bovet, I. Furno, A. Fasoli, K. Gustafson, and P. Ricci, Plasma Phys. Controlled Fusion 55, 124021 (2013).

[19] A. Bovet, M. Gamarino, I. Furno, P. Ricci, A. Fasoli, K. Gustafson, D. E. Newman, and R. Sánchez, Nucl. Fusion 54, 104009 (2014).

[20] H. Johnsen, H. L. Pécseli, and J. Trulsen, Phys. Fluids 30, 2239 (1987).

[21] I. Furno, B. Labit, A. Fasoli, F. M. Poli, P. Ricci, C. Theiler, S. Brunner, A. Diallo, J. P. Graves, M. Podesta, and S. H. Muller, Phys. Plasmas 15, 055903 (2008).

[22] P. Ricci and B. N. Rogers, Phys. Plasmas 16, 092307 (2009).

[23] P. Ricci and B. N. Rogers, Phys. Rev. Lett. 104, 145001 (2010).

[24] I. Furno, B. Labit, M. Podestà, A. Fasoli, S. H. Müller, F. M. Poli, P. Ricci, C. Theiler, S. Brunner, A. Diallo, and J. Graves, Phys. Rev. Lett. 100, 055004 (2008).

[25] C. Theiler, I. Furno, P. Ricci, A. Fasoli, B. Labit, S. H. Müller, and G. Plyushchev, Phys. Rev. Lett. 103, 065001 (2009).

[26] C. Theiler, I. Furno, A. Kuenlin, P. Marmillod, and A. Fasoli, Rev. Sci. Instrum. 82, 013504 (2011).

[27] S. H. Müller, A. Fasoli, B. Labit, M. McGrath, O. Pisaturo, G. Plyushchev, M. Podestà, and F. M. Poli, Phys. Plasmas 12, 090906 (2005).

[28] See Supplemental Material at http://link.aps.org/ supplemental/10.1103/PhysRevLett.113.225001 for details.

[29] I. Furno, M. Spolaore, C. Theiler, N. Vianello, R. Cavazzana, and A. Fasoli, Phys. Rev. Lett. 106, 245001 (2011).

[30] C. Theiler, I. Furno, A. Fasoli, P. Ricci, B. Labit, and D. Iraji, Phys. Plasmas 18, 055901 (2011). 